\title{
IN SEARCH OF AN ARTIFICIAL MORPHOTROPIC PHASE BOUNDARY: LEAD FREE BARIUM TITANATE BASED COMPOSITES
}

\author{
S. Balčiūnas ${ }^{a}$, M. Ivanov ${ }^{a}$, J. Banys ${ }^{a}$, S. Ueno ${ }^{b}$, and S. Wada ${ }^{b}$ \\ ${ }^{a}$ Faculty of Physics, Vilnius University, Sauletekio 9-III, 10222 Vilnius, Lithuania \\ ${ }^{\mathrm{b}}$ Integrated Graduate School of Medicine, Engineering and Agricultural Sciences, University of Yamanashi, \\ Kofu, Yamanashi 400-8510, Japan \\ Email: sergejus.balciunas@ff.vu.lt
}

Received 3 September 2020; revised 27 October 2020; accepted 27 October 2020

\begin{abstract}
Broadband dielectric spectroscopy was used to study potassium niobate (KN) and barium titanate (BT) coreshell-like composites. KN shell was obtained using a solvothermal reaction method. High frequency results show a huge anomaly that is associated with electro-mechanical coupling. We tried to extract spontaneous polarization values using a theoretical model. We also observed little or no evidence of KN-related phase transitions in the dielectric spectra, although infrared measurements showed typical $\mathrm{KN}$ spectral features. Furthermore, we show that a small change in the sintering process allows tuning of dielectric permittivity by an order of magnitude.
\end{abstract}

Keywords: ferroelectricity, barium titanate, core-shell, composites, dielectric measurements, electromechanical resonance

\section{Introduction}

Piezoelectric materials play an important role in our everyday electronic devices. $\mathrm{Pb}\left(\mathrm{Zr}_{x} \mathrm{Ti}_{1-x}\right) \mathrm{O}_{3}$ $(\mathrm{PZT})$ is one of the most widely used piezoelectric materials due to its high dielectric and piezoelectric constant and ability to operate in a wide temperature range [1]. In last few decades, numerous studies showed a high toxicity of lead to the environment and health [2-4. New restrictions are formed on the national level all over the world, limiting the use of lead in certain fields [5, 6]. As a result, a lot of research on lead free piezoelectric solid solutions such as $\mathrm{Na}_{x} \mathrm{Bi}_{1-x} \mathrm{TiO}_{3}, \mathrm{~K}_{x} \mathrm{Na}_{x-1} \mathrm{NbO}_{3}$, $\mathrm{Bi}_{x} \mathrm{Na}_{1-x} \mathrm{TiO}_{3}, \quad y \mathrm{Bi}_{x} \mathrm{Na}_{1-x} \mathrm{TiO}_{3}-(1-y) \mathrm{BaTiO}_{3}$ and $(1-x) \mathrm{Ba}\left(\mathrm{Zr}_{0.2} \mathrm{Ti}_{0.8}\right) \mathrm{O}_{3}-x\left(\mathrm{Ba}_{0.7} \mathrm{Ca}_{0.3}\right) \mathrm{TiO}_{3}$ [7-11] has been done. Yet, compared to PZT solid solutions, their dielectric and piezoelectric properties are still poor. As reported by others [12, 13], the nature of PZT exceptional properties is related to the morphotropic phase boundary (MPB). MPB is a structural phase transition due to the change of composition. In this region, an increase in piezoelectric properties is often observed. Current research on lead free piezoelectric materials is focused on preparing systems with MPB. In this case, both compositions must dissolve in each other to create a solid solution, and not all MPBs exhibit superior piezoelectric properties. However, a different approach can be used, that allows a much broader selection in compositions, where it is not required that compounds dissolve 
in each other. It is possible to create similar conditions as in MPB by forming a bulk hetero-epitaxial interphase [14, 15]. In such system, a thin layer of a shell compound surrounds and stresses the lattice of a core compound that may result in improved overall properties. In this paper, we show a composite structure, where the core phase is stressed by the shell phase (a layer, deposited onto the structure formed by core material). Our study is focused on improving the ferroelectrical properties of barium titanate $\mathrm{BaTiO}_{3}$ (BT) by creating stress with the potassium niobate $\mathrm{KNbO}_{3}(\mathrm{KN})$ shell.

\section{Materials and methods}

In this study, BT, $0.22 \mathrm{KNBT}$ and $0.25 \mathrm{KNBT}$ samples (the number refers to KN/BT molar ratio) were prepared in the following steps. The tetragonal BT powders $\left(\mathrm{BaTiO}_{3}\right.$, particle size of approximately $300 \mathrm{~nm}$, Sakai Chemical Industry) were used. The $\mathrm{BaTiO}_{3}$ powders were mixed with ethanol and an organic binder, poly(vinyl butyral) (the average polymerization degree of 1000, Wako Pure Chemical Industries), making $2 \mathrm{wt} \%$ of $\mathrm{BaTiO}_{3}$ powders, and dried at room temperature. The resultant powders were sieved and pressed into a green compact using a uniaxial press at $250 \mathrm{MPa}$. The binder was burned out at $600^{\circ} \mathrm{C}$ for $10 \mathrm{~h}$. To promote necking of $\mathrm{BaTiO}_{3}$ particles, these compacts were sintered at $1000^{\circ} \mathrm{C}$ for $2 \mathrm{~h}$ in air. The $\mathrm{BaTiO}_{3}$ compacts with a necking structure were immersed in $5.2 \mathrm{M}$ niobium pentaethoxide $\left(\mathrm{Nb}\left(\mathrm{OC}_{2} \mathrm{H}_{5}\right)_{5}, 99.99 \%\right.$, Kojundo Chemical Laboratory) ethanol (EL grade, Kanto Chemical) solution in $\mathrm{N}_{2}$ atmosphere. These compacts were then immersed in water at $20^{\circ} \mathrm{C}$ for $6 \mathrm{~h}$ to facilitate hydrolysis and condensation of $\mathrm{Nb}\left(\mathrm{OC}_{2} \mathrm{H}_{5}\right)_{5}$, and niobium hydroxide gels were formed inside the $\mathrm{BaTiO}_{3}$ compacts. After drying at room temperature, the $\mathrm{BaTiO}_{3}$ compacts with niobium hydroxide gels were heated at $600^{\circ} \mathrm{C}$ for $5 \mathrm{~h}$. The amount of the $\mathrm{Nb}$ source added to the $\mathrm{BaTiO}_{3}$ compacts was controlled by the number of cycles of the immersion and the subsequent heating procedures. The resultant niobium oxide/BT compact was placed in a Teflon container filled with $\mathrm{KOH}$ (UGR grade, Kanoto Chemical) and $\mathrm{K}_{2} \mathrm{CO}_{3}$ (UGR grade, Rare Metallic) ethanolic solution $(\mathrm{KOH} /$ $\left.\mathrm{K}_{2} \mathrm{CO}_{3}=0.22,\left[\mathrm{~K}^{+}\right]=1.0 \mathrm{M}\right)$. The $\mathrm{K} / \mathrm{Nb}$ atomic ratio was fixed to 10 . Then, the solvothermal treatment was carried out at $230^{\circ} \mathrm{C}$ for $20 \mathrm{~h}$ in an autoclave.
After the solvothermal treatment, the compacts were washed with ethanol and dried at $200^{\circ} \mathrm{C}$ for $5 \mathrm{~h}$. The composition ratio of the obtained KN/BT complex ceramics was calculated based on mass changes due to the complete conversion reaction of $\mathrm{Nb}_{2} \mathrm{O}_{5}$ into $\mathrm{KN}$. In this study, we prepared the KN/ BT complex ceramics with the composition ratio of $\mathrm{KN} / \mathrm{BT}=0.22$ and $\mathrm{KN} / \mathrm{BT}=0.25$, which were obtained by repeating for 3 and 5 cycles of the immersion and the subsequent heating procedures, and subsequently these samples were named through this manuscript as $0.22 \mathrm{KNBT}$ and $0.25 \mathrm{KNBT}$, respectively. We also investigated the sintered $\mathrm{BaTiO}_{3}$ (BT) compact with a necking structure as a reference sample. Additional information can be found elsewhere [14, 15].

Dielectric measurements were performed at $100-500 \mathrm{~K}$ temperature and in the $10^{2}-10^{11} \mathrm{~Hz}$ frequency range. The samples were polished and washed in acetone using an ultrasonic bath, and then parallel electrodes were made using silver paste. At the lowest frequencies $\left(20-10^{6} \mathrm{~Hz}\right)$, a flat capacitor method and a precision LCR meter HP4284A were employed. For higher frequency data $\left(10^{6}-10^{9} \mathrm{~Hz}\right)$, a coaxial line was terminated by a flat capacitor - the complex reflection coefficient was measured using an Agilent 8714ET vector network analyzer. And for highest frequencies, dielectric rods were made. The samples were placed in a waveguide system, and then reflection and transmission were measured using an Elmika scalar network analyzer R2400. Dielectric permittivity was obtained by solving the optimization problem. Dielectric hysteresis was obtained using an aixACCT TF Analyzer 2000E system. All measurements were performed during the cooling cycle at $1 \mathrm{~K} / \mathrm{min}$ rate, with a T type thermocouple or a PT100 temperature sensor.

\section{Dielectric spectra}

Dielectric measurements of BT, $0.22 \mathrm{KNBT}$ and $0.25 \mathrm{KNBT}$ systems were performed. Temperature dependences of $0.25 \mathrm{KNBT}$ at different frequencies are shown in Fig. 11. Here, three anomalies are observed and these anomalies coincide with the phase transitions in barium titanate at temperatures of 203, 278 and $393 \mathrm{~K}$ [17]. However, the same results show no sign of potassium niobate phase transitions at temperatures of 263 and $481 \mathrm{~K}$, although 


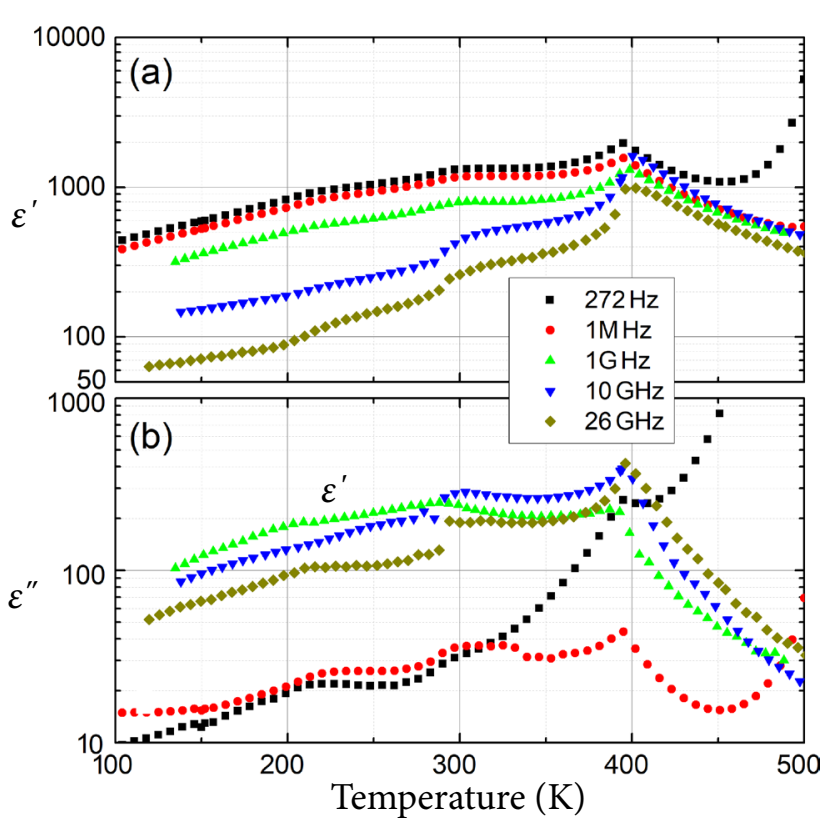

Fig. 1. Temperature dependence of the real (a) and imaginary (b) parts of dielectric permittivity for composite $0.25 \mathrm{KNBT}$.

infrared measurements showed the evidence of $\mathrm{KN}$ phase (see Fig. A1 in the Appendix).

There could be several reasons why we have a low contribution to dielectric permittivity from the KN phase. First of all, KN could have a high concentration of defects as it was obtained during the solvothermal reaction [1 8]. Moreover, the crystallite sizes of $\mathrm{KN}$ are around $50 \mathrm{~nm}$ and, considering the size effect, the dielectric permittivity should be low [19]. Thus, the phase transitions might be screened by extrinsic effects.

Furthermore, frequency dependences of complex dielectric permittivity (Fig. 2) were approximated using the superposition of several ColeCole functions [20]:

$$
\varepsilon^{*}=\varepsilon_{\infty}+\sum_{n=1,2} \frac{\Delta \varepsilon_{n}}{1+\left(\mathrm{i} \omega \tau_{n}\right)^{\alpha_{n}}} .
$$

Here, $\varepsilon_{\infty}$ is high frequency dielectric permittivity, $\tau$ is mean relaxation time, $\omega$ is frequency, $\Delta \varepsilon$ is contribution to dielectric permittivity, and $\alpha$ is parameter determining the breadth of the distribution of relaxation times $(\alpha=1$ means $\delta$-function type distribution, $\alpha=0$ means infinitely broad distribution).

The results show one clear relaxation with relaxation time of $60 \pm 12$ ps. This relaxation gives the main contribution to dielectric permittivity.

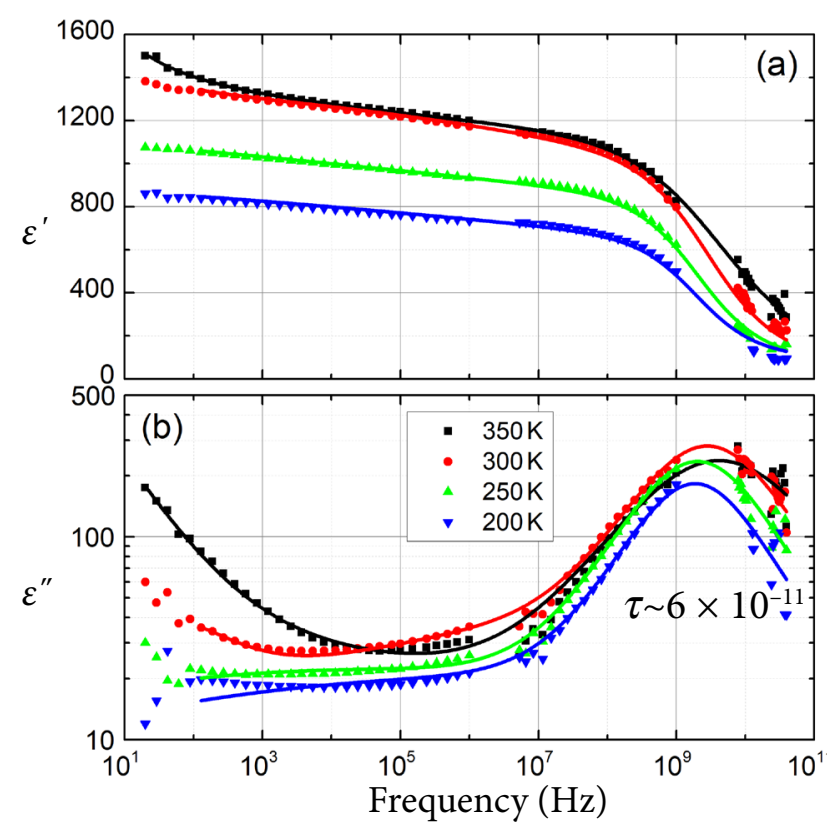

Fig. 2. Frequency dependence of the real (a) and imaginary (b) parts of dielectric permittivity for $0.25 \mathrm{KNBT}$.

The fact that this relaxation time hardly depends on temperature strongly suggests that the nature is related to acoustic resonance. In short, as the ferroelectric domain wall moves, it emits Barkhausen acoustic noise [21], which can resonate in the grain or in a grain cluster; as BT is piezoelectric, the response can be seen in dielectric spectra. Alternatively, a uniformly polarized grain can contribute to the dielectric permittivity via electromechanical coupling, with contribution determined by characteristics of the acoustic resonance of the whole grain. Assuming the electromechanical mechanism, it is possible to calculate the acoustic wavelength from the experimental data. It is reported that the velocity of acoustic waves in BT is around $5.5 \mathrm{~km} / \mathrm{s}$ [22. 23. The calculated half wavelength is $1030 \pm 200 \mathrm{~nm}$ that is in a good agreement with the BT cluster size in the $0.25 \mathrm{KNBT}$ system (see Fig. A2 in the Appendix). Interestingly, this contribution disappears at temperatures above the Curie point, as can be seen from Fig. 1 (only relaxation due to Maxwell-Wagner polarisation remains above $400 \mathrm{~K}$ ). The same happens with the broad relaxation in frequencies of $10^{3}-10^{6} \mathrm{~Hz}$. This could indicate that the source of the latter relaxation is domain wall motion in BT grains. Similar measurements were done with the core-shell samples where BT was replaced with strontium titanate (ST). In this case, the dielectric data showed no dispersions up to $30 \mathrm{GHz}$ (see 
Fig. A3 in the Appendix). This further supports our idea of an electromechanical origin of this microwave process observed in KNBT composites.

Moreover, the $\mathrm{BT}$ and $0.25 \mathrm{KNBT}$ composites have a similar dielectric permittivity, although the densities are quite different (BT 59.53\% and $0.25 \mathrm{KNBT} 78.67 \%$ ). This fact further supports our assumption that $\mathrm{KN}$ gives almost no contribution to dielectric permittivity, as the core structure of $0.25 \mathrm{KNBT}$ was similar to the pure BT pellet. The main difference between the experimental data is a higher conductivity for the $0.25 \mathrm{KNBT}$ system at high (above $450 \mathrm{~K}$ ) temperatures that could be due to defects in the KN structure. Also, a significantly lower dielectric permittivity was observed in the $0.22 \mathrm{KNBT}$ system, that cannot be explained by a lower density or low KN contribution (Fig. 3).

The $0.22 \mathrm{KNBT}$ and $0.25 \mathrm{KNBT}$ have different BT structures (see Fig. A4 in the Appendix). The $0.22 \mathrm{KNBT}$ composite has a necking connection between grains, and the grains of $0.25 \mathrm{KNBT}$ composite are merged into bigger clusters. Lower dielectric permittivity can be explained using an effective medium approach, i.e. the Lichtenecker model [24, 25]:

$$
\varepsilon^{* \beta}=X \varepsilon_{1}^{\beta}+(1-X) \varepsilon_{2}^{\beta},-1 \leq \beta \leq 1 .
$$

Here, $\varepsilon^{*}$ is effective complex dielectric permittivity, $X$ is volume fraction of the first medium, $\varepsilon_{1}, \varepsilon_{2}$ are dielectric permittivity of media one and two, respectively, and $\beta$ is parameter describing the struc-

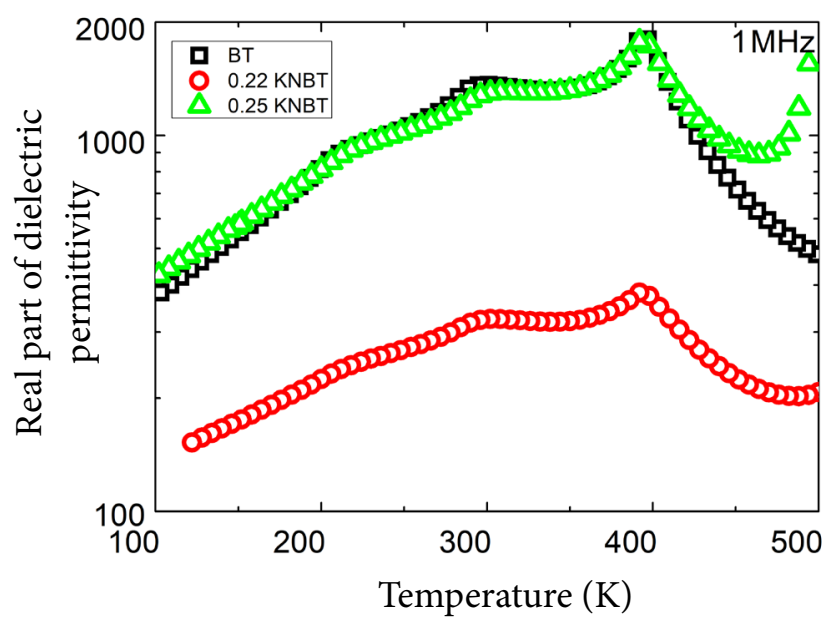

Fig. 3. Temperature dependence of the real part of dielectric permittivity for BT, $0.22 \mathrm{KNBT}$ and $0.25 \mathrm{KNBT}$ at $1 \mathrm{MHz}$ frequency. ture of the system: if $\beta=-1$, the system is made of plates connected in series, and if $\beta=1$, it is made of parallel pillars (see Fig. A5 in the Appendix). In the $0.22 \mathrm{KNBT}$ and $0.25 \mathrm{KNBT}$ systems, both phases percolate, meaning $\beta>0$ [25]. Also, longer calcination time results in systems with larger interconnections between grains and a more pillarlike structure than a necking system, this means that parameter $\beta$ is higher for $0.25 \mathrm{KNBT}$ than for $0.22 \mathrm{KNBT}$. It can be mathematically shown that for smaller $\beta$ values the phase with lower dielectric permittivity becomes dominant [25].

\section{Polarization}

The acoustic resonance causes a change in polarization, which gives a rise in dielectric permittivity. Arlt et al. have calculated the relationship between spontaneous polarization and contribution to dielectric permittivity in the presence of ferro-elastic domains [26]:

$$
\Delta \varepsilon_{\mathrm{r}}=\frac{P_{0}^{2}}{2 C_{55} \varepsilon_{0} S_{0}^{2}}, f_{\mathrm{c}}=\frac{\sqrt{C_{55} / q}}{\pi d} .
$$

Here, $P_{0}$ is spontaneous polarization, $C_{55}$ is (shear) elastic constant, $\varepsilon_{0}$ is vacuum dielectric constant, $S_{0}$ is spontaneous deformation, $\rho$ is density, and $f_{c}$ is mean relaxation frequency. An effective contribution to dielectric permittivity $\Delta \varepsilon$ was obtained from Cole-Cole approximations (Fig. 2). To obtain real dielectric permittivity $\Delta \varepsilon_{\mathrm{r}}$ values from the experimental effective ones, we applied the Lichtenecker model, with $\beta=0.4$ [27]. It is worth noting that the permittivity $\varepsilon_{1}\left(\Delta \varepsilon_{\mathrm{r}}=\varepsilon_{1}\right)$ of the first phase (BT) is a lot higher than that of the second one (air or composite of air and $\mathrm{KN}$ ). In this case, we can assume that the dielectric permittivity of the second phase is infinitesimal and can be neglected:

$$
\varepsilon_{1}=\left(\frac{\varepsilon^{* \beta}-(1-X) \varepsilon_{2}^{\beta}}{X}\right)^{\frac{1}{\beta}} \cong \frac{\varepsilon^{* \beta}}{X^{1 / \beta}} .
$$

The obtained evaluation of the real permittivity of core material allows us to apply Eq. (3). In our work, we replace elastic constant $\mathrm{C}_{55}$ with more general Young modulus as in our case we have homogeneous ceramic and resonance occurring in grain clusters which are randomly orientated. Temperature dependence of Young modules was taken from [28], electrostriction coefficient Q from [29, 30]. 
Spontaneous deformation can be written as $S_{0}=P_{0}^{2} \mathrm{Q}$ [26]. Using Arlt's model we have managed to obtain the spontaneous polarization temperature dependence curve that is similar to the experimental curve found in literature [31] (Fig. 4).

However, in our case calculations were based on ceramic measurements and due to the random rotation orientation of grains, the calculated polarization should be around two times lower than in crystals. It is reported that at room temperature pure, high quality BT crystals have polarization values up to $26-27 \mu \mathrm{C} / \mathrm{cm}^{2}$ [26, 32, 33], that is still higher than those obtained here by around $15-30 \%$ (the double value of the obtained polarization at room temperature is $19-23 \mu \mathrm{C} / \mathrm{cm}^{2}$ ).

In order to verify our calculations, hysteresis loops measurements were performed in vacuum (pressure $<10^{-4} \mathrm{hPa}$ ) with $100 \mathrm{kV} / \mathrm{cm}$ field for the $0.25 \mathrm{KNBT}$ and BT systems (see Fig. A6 in the Appendix), which possess the same structure of the core material. The results showed slim hysteresis loops with high back switching and low remnant polarization values $(4 \mu \mathrm{C} / \mathrm{cm})$ (Fig. 4), typical of fine grain ceramics [34, 35]. Furthermore, the $P(E)$ measurements showed high coercive field up to $10 \mathrm{kV} / \mathrm{cm}$, that suggests about a high number of deep defects in the BT structure, which may pin the domain walls. This could explain different results obtained from the calculation and $P(E)$ measurements. Dielectric measurements are done with small amplitude signals, this means that the domain wall moves just slightly, and if the domain wall is pinned at some point, other points of the wall can still move. We can imagine the domain wall as a membrane. The mobile sections of domain walls have a small chance to move far enough to encounter a new pinning defect. On the other hand, for $P(E)$ measurements high fields are applied, in this case domain walls have to move huge distances; and if a domain wall is pinned, deep defects force the domain wall to move back after the removal of electrical field, resulting in a low apparent remnant polarization [35]. In such case, very large fields, which are enough to unpin domain walls, are needed to estimate the true spontaneous polarization. Thus, the indirect method to estimate the switchable polarization based on dielectric spectroscopy has an advantage of being less sensitive to pinning defects.

In addition, we observe higher spontaneous polarization values for the $0.25 \mathrm{KNBT}$ system in both cases. There could be several explanations. First of all, our calculation shows a lower polarization value

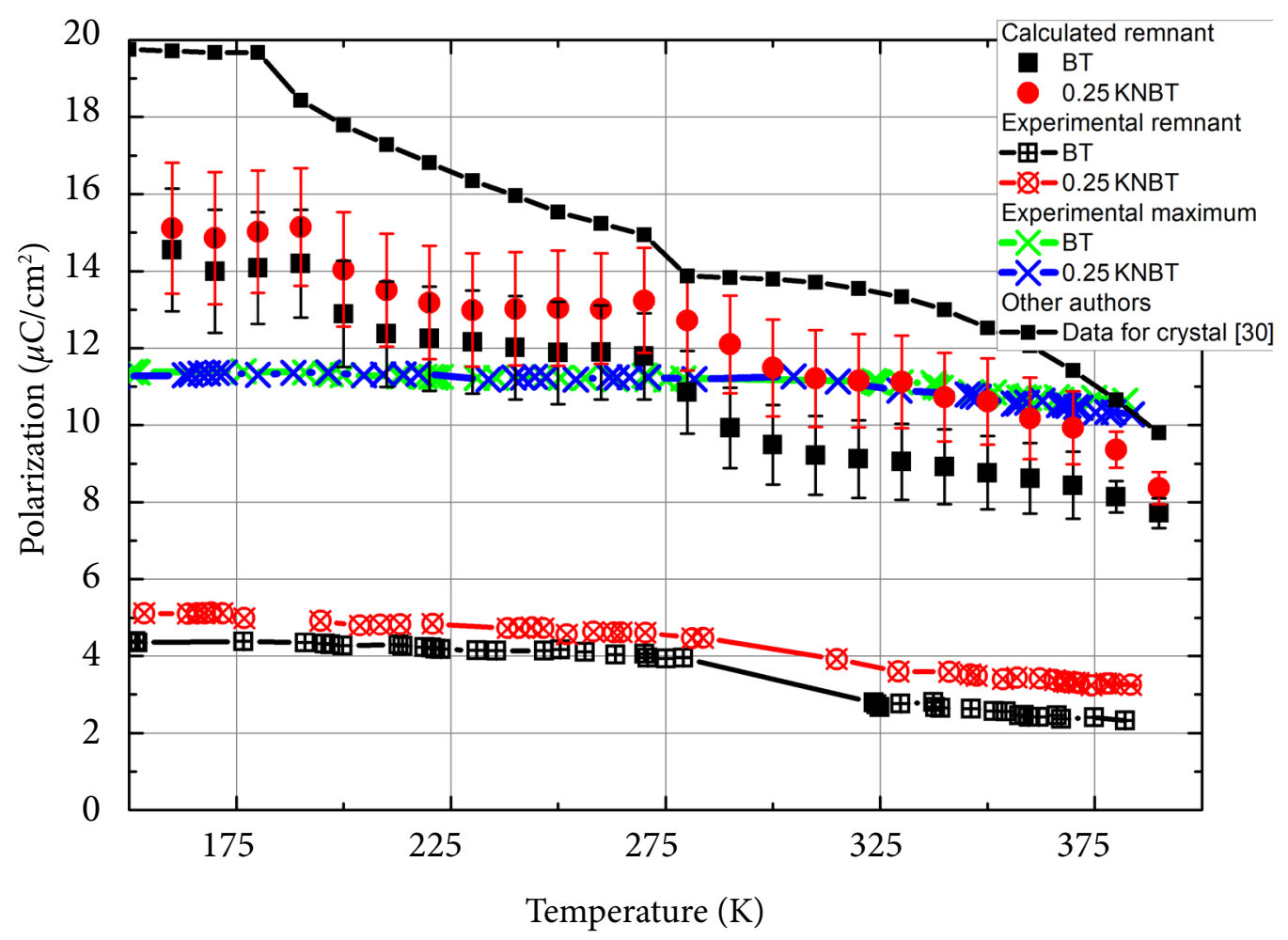

Fig. 4. Calculated and measured spontaneous polarization values for BT and KNBT systems. The error bars for measured systems are smaller than experimental points. 
than that found in literature; this could mean that there are some domain walls that cannot move at all, for instance, the domain wall can be fixed to the BT grain boundary surface. If we deposit a film of $\mathrm{KN}$ on the BT grain, we decrease surface tension on BT grains; also, KN could remove the absorbed matter on BT. All in all, we change the grain boundary condition on the BT surface and some domain walls could be unpinned. This theory works for the results obtained from $P(E)$ measurements as remnant polarization is increased by a fixed amount in all temperature range. For the calculated polarization results, however, it is observed that the spontaneous polarization of $0.25 \mathrm{KNBT}$ at temperatures up to $278 \mathrm{~K}$ is equal to that of $\mathrm{BT}$ in the margin of error, but above that temperature the spontaneous polarization of $0.25 \mathrm{KNBT}$ is higher. At $278 \mathrm{~K}$ temperature a phase transition, from orthorhombic (lower temperature phase) to tetragonal, occurs in pure BT. Thus, the second explanation could follow: the epitaxial $\mathrm{KN}$ on the BT surface stresses the BT microscopic structure (especially in the tetragonal phase), leading to an increase in the domain wall count and spontaneous polarization, similarly as in the systems with a morphotropic phase boundary [14]. Possibly, the third explanation should be considered: according to Ref. [36], enhanced non- $180^{\circ}$ switching was observed in the KN-BT composites, which could be the reason of an easy movement of the domain walls in a low signal regime. Thus, we obtain a higher contribution to dielectric permittivity, and from Eq. (3) a higher switchable spontaneous polarization. This explanation seems favourable also due to the fact that at high fields polarization is the same in both $0.25 \mathrm{KNBT}$ and pure BT, meaning that the value of spontaneous polarization has not changed. Interestingly, the high-field value is very similar to that obtained from dielectric data, so the estimate is correct. However, the KN shell improves the mobility of domain walls, leading to an enhanced electromechanical response. This, in a sense, is the artificial MPB.

\section{Conclusions}

Based on the dielectric spectroscopy results, it can be concluded that the main contribution to dielectric permittivity in the microwave region comes from the electro-mechanical resonance in BT grains. Lower dielectric permittivity values in the $0.22 \mathrm{KNBT}$ system were observed due to dif- ferent BT structure topology. Thus, controlling BT structure sizes and topology in the sample during the preparation stage allows us to tune the dielectric response of our system. Also, the same results show almost no contribution to dielectric permittivity from $\mathrm{KN}$ in the $0.25 \mathrm{KNBT}$ system.

And lastly, remnant polarization values for the composite $0.25 \mathrm{KNBT}$ are higher than for the pure BT. Several reasons could follow. Firstly, KN on BT grains reduces the surface tension or absorbed matter and reduces the deep defect count which allows an easier domain wall movement. Secondly, KN stresses the BT microscopic structure, thus increasing the domain wall count similarly as at the morphotropic phase boundary. Even though the first explanation cannot be excluded, it is not sufficient. Thus, the second mechanism is needed to explain some of the observed experimental results. This can be considered an indication of an artificial MPB.

\section{Acknowledgements}

This research was funded by a Grant (No. LJB3/2016) from the Research Council of Lithuania. This research was performed in cooperation with the University of Yamanashi.

\section{Appendix}

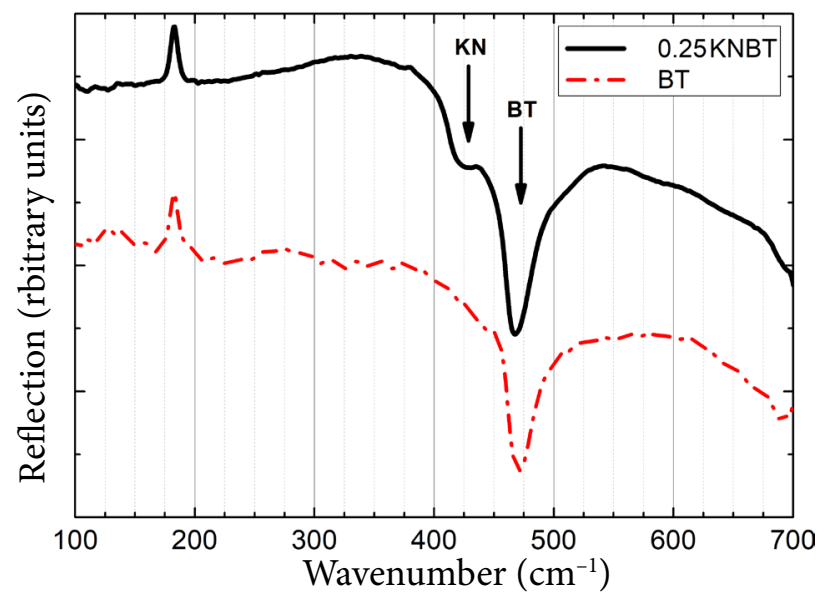

Fig. A1. Infrared spectra show the evidence of potassium niobate $(\mathrm{KN})$ phase in the $0.25 \mathrm{KNBT}$ system. The dot-dashed (red online) curve represents pure barium titanate (BT) samples. The frequencies of all phonon modes coincide well with works published earlier [37]. The black curve shows one additional phonon mode which represents the KN phonon mode at $430 \mathrm{~cm}^{-1}$ [38]. A small gradual reflection intensity decrease at higher frequencies is due to porous samples. 


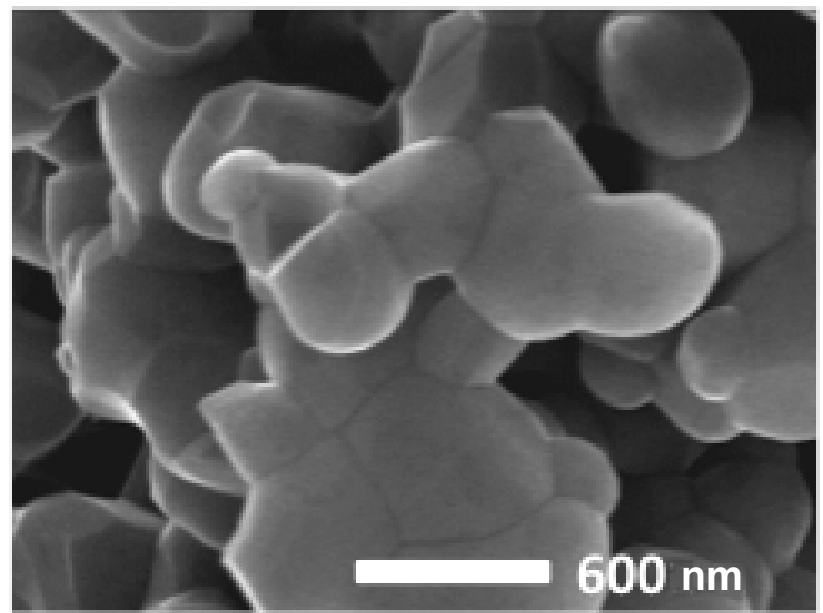

Fig. A2. SEM image of the pure BT structure shows closely packed clusters of BT particles. The sizes of clusters are around 1 micron in diameter. In this work there were 2 samples with this structure: pure BT and KNBT0.25. To obtain KNBT0.25 we submerged pure BT pellets into ethanol, $\mathrm{KOH}, \mathrm{K}_{2} \mathrm{CO}_{3}$ and $\mathrm{Nb}_{2} \mathrm{O}_{5}$ solution for solvothermal solidification reactions.

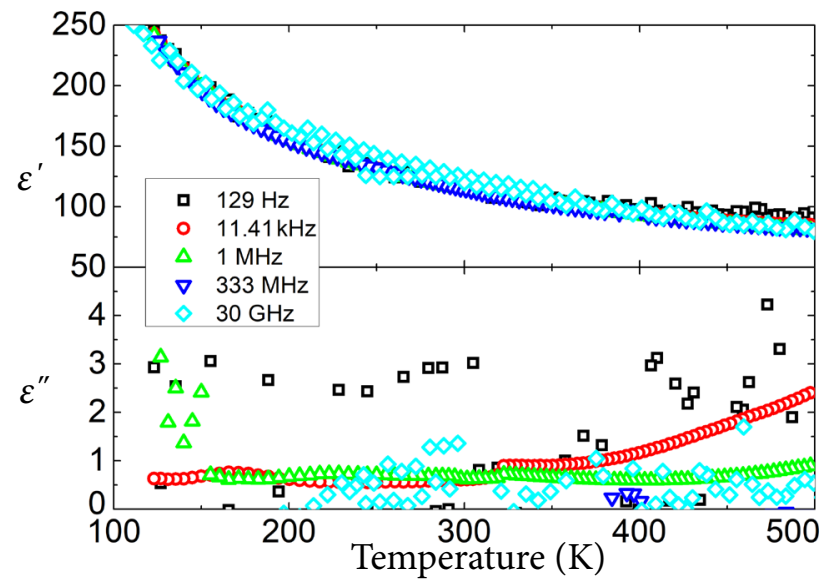

Fig. A3. Temperature dependences of $\varepsilon^{\prime}$ of $0.25 \mathrm{KNST}$ system at different frequencies are on the same curve. This means that we have no dispersion in all measured temperature range. These results supplement our earlier statement that there must be piezoelectric grains for electrometrical resonance to occur.

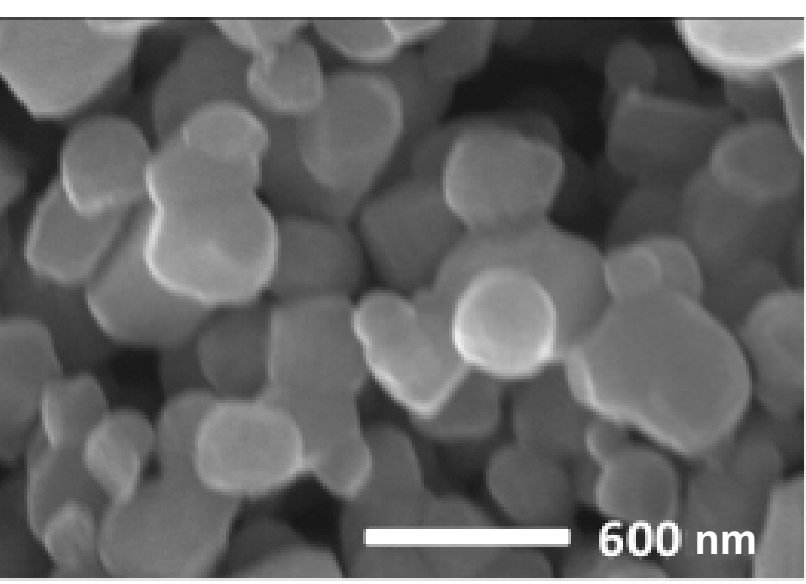

Fig. A4. SEM image of the pure BT shows a necking BT structure. Cluster sizes are around 500-600 nm. In this work one sample with this structure is presented: KNBT0.22. This sample is prepared similarly as KNBT0.25 shown earlier.
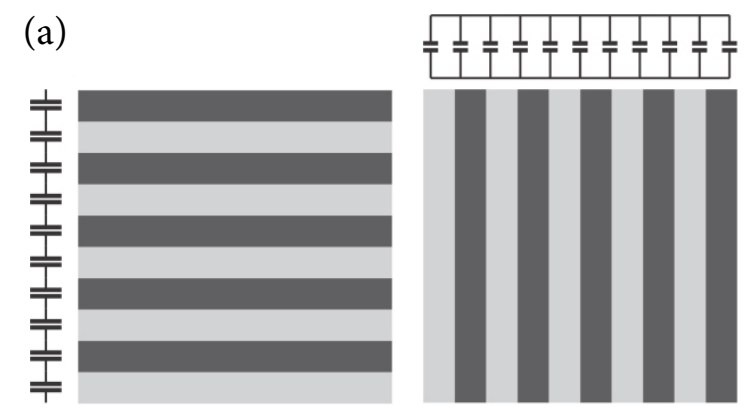

(b)
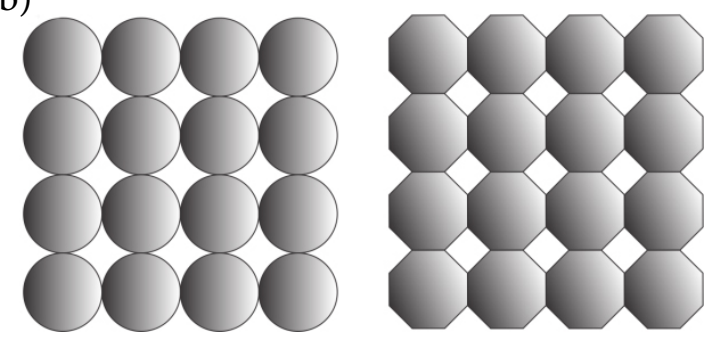

Fig. A5. Coarse approximation of the Lichtenecker model. $\beta$ is a parameter describing the structure of the system: if $\beta=-1$, the system is made of plates connected in series, and if $\beta=1$, it is made of parallel pillars (a). In our case we have a complex intermediate structure. In (b) we have compared two systems with unbaked (left) and baked (right) grains. We observe that the baked system resembles a connected pillarlike structure. 


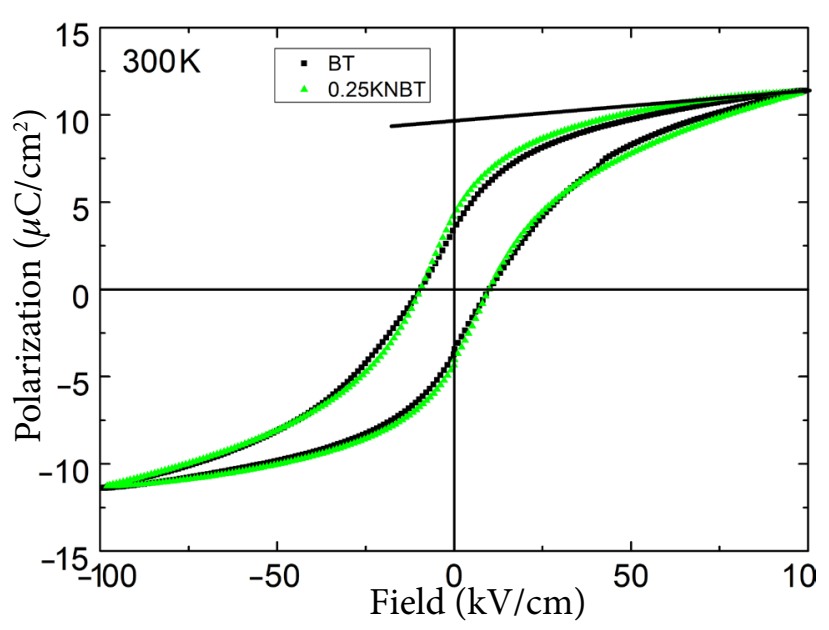

Fig. A6. $P(E)$ measurements for pure BT and $0.25 \mathrm{KNBT}$ structures. Both structures show similar saturation polarization values at room temperature which are around $11.3 \mu \mathrm{C} / \mathrm{cm}^{2}$. Also high back switching is observed which is typical of fine grain ceramics. Alternative high back switching could occur due to a large amount of defects [18]. Furthermore, we have observed that samples with KN have a higher remnant polarization value, which is around $4.4 \mu \mathrm{C} / \mathrm{cm}^{2}$, and values for pure BT are $3.2 \mu \mathrm{C} / \mathrm{cm}^{2}$.

\section{References}

[1] D. Berlincourt, C. Cmolik, and H. Jaffe, Piezoelectric properties of polycrystalline lead titanate zirconate compositions, Proc. IRE 48(2), 220-229 (1960).

[2] G. Ngueta and A.P. Kengne, Low-level environmental lead exposure and dysglycemia in adult individuals: Results from the Canadian health and measure survey 2007-2011, Biol. Trace Elem. Res. 175(2), 278-286 (2017).

[3] S. Tong, Y.E. von Schirnding, and T. Prapamontol, Environmental lead exposure: a public health problem of global dimensions, Bull. World Health Organ. 78(9), 1068-1077 (2000).

[4] M.D. Sanborn, A. Abelsohn, M. Campbell, and E. Weir, Identifying and managing adverse environmental health effects: 3. Lead exposure, Can. Med. Assoc. J. 166(10), 1287-1292 (2002).

[5] E.U. Council, Directive 2002/95/EC of the European Parliament and of the Council, https://eur-lex.europa.eu (2003).
[6] U.S. EPA, Summary of the Toxic Substances Control Act, https://www.epa.gov (1976).

[7] Y. Qin, S. Zhang, Y. Wu, C. Lu, and J. Zhang, Impacts of acceptor doping on the piezoelectric properties and domain structure in NBT-based lead-free ceramics, J. Eur. Ceram. Soc. 37(11), 3493-3500 (2017).

[8] T. Zheng, H. Wu, Y. Yuan, X. Lv, Q. Li, T.-L. Men, C. Zhao, D. Xiao, J. Wu, K. Wang, et al., The structural origin of enhanced piezoelectric performance and stability in lead free ceramics, Energy Environ. Sci. 10(2), 528-537 (2017), https://doi. org/10.1039/C6EE03597C

[9] G. Viola, R. McKinnon, V. Koval, A. Adomkevicius, S. Dunn, and H. Yan, Lithium-induced phase transitions in lead-free $\mathrm{Bi}_{0.5} \mathrm{Na}_{0.5} \mathrm{TiO}_{3}$ based ceramics, J. Phys. Chem. C 118(16), 8564-8570 (2014), https://doi.org/10.1021/jp500609h

[10]W. Bai, P. Li, L. Li, J. Zhang, B. Shen, and J. Zhai, Structure evolution and large strain response in BNT-BT lead-free piezoceramics modified with $\mathrm{Bi}\left(\mathrm{Ni}_{0.5} \mathrm{Ti}_{0.5}\right) \mathrm{O}_{3}$, J. Alloys Compd. 649 (SupplementC), 772-781 (2015), https://doi.org/10.1016/j. jallcom.2015.07.178

[11]P. Berik, W.-Y. Chang, and X. Jiang, Piezoelectric $d_{36}$ in-plane shear-mode of lead-free BZTBCT single crystals for torsion actuation, Appl. Phys. Lett. 110(5), 052902 (2017), https://doi. org/10.1063/1.4975587

[12]B. Jaffe, Piezoelectric Ceramics (Elsevier, 2012).

[13]R. Guo, L. Cross, S. Park, B. Noheda, D. Cox, and G. Shirane, Origin of the high piezoelectric response in $\mathrm{PbZr}_{1-x} \mathrm{Ti}_{x} \mathrm{O}_{3}$, Phys. Rev. Lett. 84(23), 5423 (2000).

[14]S. Wada, S. Shimizu, K. Yamashita, I. Fujii, K. Nakashima, N. Kumada, Y. Kuroiwa, Y. Fujikawa, D. Tanaka, and M. Furukawa, Preparation of barium titanate-potassium niobate nanostructured ceramics with artificial morphotropic phase boundary structure by solvothermal method, Jpn. J. Appl. Phys. 50(9S2), 09NC08 (2011).

[15]I. Fujii, S. Shimizu, K. Yamashita, K. Nakashima, N. Kumada, C. Moriyoshi, Y. Kuroiwa, Y. Fujikawa, D. Tanaka, M. Furukawa, and S. Wada, Enhanced piezoelectric response of $\mathrm{BaTiO}_{3}-\mathrm{KNbO}_{3}$ 
composites, Appl. Phys. Lett. 99(20), 202902 (2011), https://doi.org/10.1063/1.3662397

[16]G.K.L. Goh, C.G. Levi, J.H. Choi, and F.F. Lange, Hydrothermal epitaxy of $\mathrm{KNbO}_{3}$ thin films and nanostructures, J. Cryst. Growth 286(2), 457-464 (2006), https://doi.org/10.1016/j.jcrysgro.2005.10.021

[17]A. von Hippel, Ferroelectricity, domain structure, and phase transitions of barium titanate, Rev. Mod. Phys. 22(3), 221 (1950).

[18]Y. Hirose, S. Ueno, K. Nakashima, and S. Wada, Preparation of $\mathrm{BaTiO}_{3}$ nano-structured ceramics by solvothermal solidification method, Trans. Mater. Res. Soc. Jpn. 40(3), 239-242 (2015).

[19]Z. Zhao, V. Buscaglia, M. Viviani, M.T. Buscaglia, L. Mitoseriu, A. Testino, M. Nygren, M. Johnsson, and P. Nanni, Grain-size effects on the ferroelectric behavior of dense nanocrystalline $\mathrm{BaTiO}_{3}$ ceramics, Phys. Rev. B 70(2), 024107 (2004).

[20]K.S. Cole and R.H. Cole, Dispersion and absorption in dielectrics I. Alternating current characteristics, J. Chem. Phys. 9(4), 341-351 (1941).

[21]R. Newton, A. Ahearn, and K. McKay, Observation of the ferro-electric Barkhausen effect in barium titanate, Phys. Rev. 75(1), 103 (1949).

[22]H.B. Huntington and R.D. Southwick, Ultrasonic velocities in polarized barium titanate ceramics, J. Acoust. Soc. Am. 27(4), 677-679 (1955), https://doi.org/10.1121/1.1907991

[23]S. Kashida, I. Hatta, A. Ikushima, and Y. Yamada, Ultrasonic velocities in $\mathrm{BaTiO}_{3}$, J. Phys. Soc. Jpn. 34(4), 997-1001 (1973), https://doi.org/10.1143/ JPSJ.34.997

[24]K. Lichtenecker, Dielectric constant of natural and synthetic mixtures, Phys. Z. 27, 115 (1926).

[25]A. Goncharenko, V. Lozovski, and E. Venger, Lichtenecker's equation: applicability and limitations, Opt. Commun. 174(1), 19-32 (2000).

[26]G. Arlt, U. Böttger, and S. Witte, Dielectric dispersion of ferroelectric ceramics and single crystals at microwave frequencies, Ann. Phys. 506(78), 578-588 (1994).

[27]D. Nuzhnyy, E. Buixaderas, I. Rychetsky, C. Kadle, J. Petzelt, H. Uršič, and B. Malič, Percolation in the dielectric function of $\mathrm{Pb}(\mathrm{Zr}, \mathrm{Ti}) \mathrm{O}_{3}-$

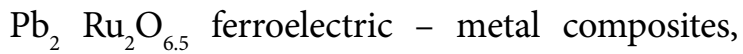

J. Phys. Appl. Phys. 47(49), 495301 (2014), https://doi.org/10.1088/0022-3727/47/49/495301

[28]B.L. Cheng, M. Gabbay, W. Duffy, and G. Fantozzi, Mechanical loss and Young's modulus associated with phase transitions in barium titanate based ceramics, J. Mater. Sci. 31(18), 4951-4955 (1996), https://doi.org/ 10.1007/BF00355886

[29]J.J. Wang, F.Y. Meng, X.Q. Ma, M.X. Xu, and L.Q. Chen, Lattice, elastic, polarization, and electrostrictive properties of $\mathrm{BaTiO}_{3}$ from firstprinciples, J. Appl. Phys. 108(3), 034107 (2010), https://doi.org/ 10.1063/1.3462441

[30]A.J. Bell, Phenomenologically derived electric field-temperature phase diagrams and piezoelectric coefficients for single crystal barium titanate under fields along different axes, J. Appl. Phys. 89(7), 3907-3914 (2001), https://doi. org/10.1063/1.1352682

[31]H.F. Kay and P. Vousden, XCV. Symmetry changes in barium titanate at low temperatures and their relation to its ferroelectric properties, Lond. Edinb. Dublin Philos. Mag. J. Sci. 40(309), 1019-1040 (1949), https://doi. org/10.1080/14786444908561371

[32] J. Shieh, J.H. Yeh, Y.C. Shu, and J.H. Yen, Hysteresis behaviors of barium titanate single crystals based on the operation of multiple $90^{\circ}$ switching systems, Mater. Sci. Eng. B 161(1), 50-54 (2009), https://doi.org/10.1016/j.mseb.2008.11.046

[33]H.H. Wieder, Electrical behavior of barium titanate single crystals at low temperatures, Phys. Rev. 99(4), 1161-1165 (1955), https://doi. org/10.1103/PhysRev.99.1161

[34]T. Schenk, E. Yurchuk, S. Mueller, U. Schroeder, S. Starschich, U. Böttger, and T. Mikolajick, About the deformation of ferroelectric hystereses, Appl. Phys. Rev. 1(4), 041103 (2014), https://doi. org/10.1063/1.4902396

[35]L. Jin, F. Li, and S. Zhang, Decoding the fingerprint of ferroelectric loops: comprehension of the material properties and structures, J. Am. Ceram. Soc. 97(1), 1-27 (2014).

[36]N.H. Khansur, H. Kawashima, S. Wada, J.M. Hudspeth, and J. Daniels, Enhanced extrinsic domain switching strain in core-shell structured $\mathrm{BaTiO}_{3}-\mathrm{KNbO}_{3}$ ceramics, Acta Mater. 
98(Supplement C), 182-189 (2015), https://doi. org/10.1016/j.actamat.2015.07.034

[37]T. Ostapchuk, J. Petzelt, M. Savinov, V. Buscaglia, and L. Mitoseriu, Grain-size effect in $\mathrm{BaTiO}_{3}$ ceramics: study by far infrared spectroscopy, Phase Transit. 79(6-7), 361-373 (2006), https://doi. org/10.1080/01411590600892047
[38]M.D. Fontana, G. Metrat, J.L. Servoin, and F. Gervais, Infrared spectroscopy in $\mathrm{KNbO}_{3}$ through the successive ferroelectric phase transitions, J. Phys. C 17(3), 483 (1984), https://doi. org/10.1088/0022-3719/17/3/020

\title{
DIRBTINĖS MORFOTROPINĖS FAZIỤ SANDŪROS BEIEŠKANT: BEŠVINIAI KOMPOZITAI BARIO TITANATO PAGRINDU
}

\author{
S. Balčiūnas ${ }^{\text {a }}$, M. Ivanov ${ }^{\text {a }}$ J. Banys ${ }^{\text {a }}$, S. Ueno ${ }^{\text {b }}$, S. Wada ${ }^{\text {b }}$ \\ ${ }^{a}$ Vilniaus universiteto Fizikos fakultetas, Vilnius, Lietuva \\ ${ }^{\mathrm{b}}$ Yamanashi universiteto Tarpdisciplinine medicinos ir inžinerijos aukštoji mokykla, Kofu, Japonija
}

\section{Santrauka}

Naudojant plačiajuostès dielektrinès spektroskopijos metodikas buvo ištirti kalio niobato (KN), bario titanato (BT) kevalo ir branduolio kompozitai. Šie kompozitai buvo susintetinti solvoterminès reakcijos metodu. Dielektriniame spektre, esant aukštiems dažniams $\left(10^{8}-10^{11} \mathrm{~Hz}\right)$, stebima didžiulè anomalija, kuri siejama su elektromechaniniu rezonansu. Nedidelis sintezès proceso pokytis gali daryti ịtaką dielektriniams spektrams, jis leidžia derinti dielektrinès skvarbos vertes. Taip pat buvo tyrinejamos spontaninès poliarizacijos vertes naudojant teorinius modelius. Galiausiai parodoma, kad kevalo ir branduolio kompozitai turi aukštesnes spontaninès poliarizacijos vertes. 